\section{RSP}

http://www.rsp.fsp.usp.br/
Revista de Saúde Pública

\title{
Infecção por HPV em mulheres atendidas pela Estratégia Saúde da Família
}

Andréia Rodrigues Gonçalves Ayres', Gulnar Azevedo e Silva", Maria Teresa Bustamante Teixeira"I', Kristiane de Castro Dias Duque III, Maria Lúcia Salim Miranda Machado'I", Carmen Justina Gamarra $^{\mathrm{IV}}$, José Eduardo Levi ${ }^{\mathrm{v}}$

I Hospital Universitário Gaffrée e Guinle. Universidade Federal do Estado do Rio de Janeiro. Rio de Janeiro, RJ, Brasil

" Departamento de Epidemiologia. Instituto de Medicina Social. Universidade do Estado do Rio de Janeiro. Rio de Janeiro, RJ, Brasil

III Núcleo de Assessoria, Treinamento e Estudos em Saúde. Universidade Federal de Juiz de Fora. Juiz de Fora, MG, Brasil

Iv Faculdade de Saúde Coletiva. Universidade Federal da Integração Latino-Americana. Foz do Iguaçu, PR, Brasil

$\checkmark$ Laboratório de Virologia. Instituto de Medicina Tropical. Universidade de São Paulo. São Paulo, SP, Brasil

\section{RESUMO}

OBJETIVO: Estimar a prevalência de infecção do colo do útero pelo HPV entre mulheres assistidas pela Estratégia Saúde da Família e identificar os fatores relacionados à infecção.

MÉTODOS: Trata-se de estudo transversal, no qual participaram 2.076 mulheres de 20 a 59 anos, residentes em Juiz de Fora, MG, convocadas para rastreamento organizado, realizado em unidades com a Estratégia Saúde da Família implantada. As participantes responderam ao questionário padronizado, realizando exame citológico cervical convencional e teste para HPV de alto risco oncogênico. Foram calculadas estimativas de prevalência de infecção pelo HPV segundo características selecionadas, referenciadas na literatura, relacionadas ao status socioeconômico, saúde reprodutiva e estilo de vida.

RESULTADOS: A prevalência global de infecção pelo HPV foi 12,6\% (IC95\% 11,16-14,05). A prevalência para o pooled primer contendo 12 tipos de HPV oncogênicos $(31,33,35,39,45$, $51,52,56,58,59,66$ e 68) foi 8,6\% (IC95\% 7,3-9,77). Na análise multivariada, observou-se que as seguintes variáveis estavam significativamente associadas a uma maior prevalência de infecção por HPV: estado conjugal (solteira: RP ajustada = 1,40; IC95\% 1,07-1,8), consumo de bebidas alcoólicas (qualquer frequência durante a vida: $\mathrm{RP}$ ajustada $=1,44$; IC95\% 1,11-1,86) e número de parceiros sexuais ao longo da vida ( $\geq 3$ : RP ajustada $=1,35$; IC95\% 1,04-1,74).

CONCLUSÕES: A prevalência de infecção pelo HPV na população estudada varia de média a particularmente alta entre as mulheres jovens. A prevalência de infecção por HPV16 e HPV18 se assemelha às mundiais. Uma distribuição homogênea entre os tipos do pooled primer precederia a infecção isolada pelo HPV18 em magnitude, podendo ser a diferença maior que a observada. A identificação da prevalência de HPV de alto risco oncogênico pode auxiliar na identificação de mulheres sob maior risco de evolução para lesão preneoplásica.

DESCRITORES: Papillomaviridae. Prevalência. Programas de Rastreamento. Neoplasias do Colo do Útero, prevenção \& controle. Estratégia Saúde da Família.

\footnotetext{
Copyright: Este é um artigo de acesso aberto distribuído sob os termos da Licença de Atribuição Creative Commons, que permite uso irrestrito, distribuição e reprodução em qualquer meio, desde que o autor e a fonte

originais sejam creditados.

Andréia Rodrigues Gonçalves Ayres e Guinle 20270-004 Rio de Janeiro, RJ, Brasi Recebido: 9 jun 2016

Como citar: Ayres ARG Duque KCD, Machado MLSM Gamarra CJ, et al. Infecção por HPV em mulheres atendidas pela Estratégia Saúde da Família. Rev Saude Publica. 2017;51:92.
} 


\section{INTRODUÇÃO}

A infecção por papilomavírus humano (HPV) é uma das infecções genitais mais frequentes no mundo e é causa necessária para ocorrência do câncer do colo do útero ${ }^{10}$. No Brasil, estima-se que 15.590 mulheres adoeçam anualmente, com taxa de incidência bruta de 15,33/100 mil, o que torna a prevenção e o controle do câncer do colo do útero prioridades nos pactos de gestão da saúde voltados para a saúde da mulher9.

A estratégia adotada globalmente é o rastreamento das lesões pré-invasivas com exame citológico cervical por esfregaço. Nos países em desenvolvimento, o impacto da introdução e da ampliação do rastreamento como política de saúde foi menor do que o observado nos países desenvolvidos, em função da organização deficiente, baixas coberturas e falta de garantia da qualidade ${ }^{21}$. Em países da América do Sul com desenvolvimento mais acelerado, observou-se um declínio na mortalidade por câncer do colo do útero, com estimativas de mudança percentual anual variando de -1,4 a -6,3 entre 1983 e 2002 ${ }^{29}$. Na América Latina, essa tendência ocorre em países com renda média mais elevada, como Argentina e Uruguai, e em países com histórico de implementação do rastreamento com maior organização, como México, Colômbia, Chile e Costa Rica, onde as taxas de mortalidade foram reduzidas quase à metade, ocasionando uma inversão na razão câncer in situ/invasivo ${ }^{19}$. No Brasil, observou-se um declínio global das taxas de mortalidade por câncer do colo do útero nas últimas décadas, porém de forma heterogênea nas distintas macrorregiões (mudança percentual anual variando de -3,3 a 1,7), mostrando um sucesso parcial ${ }^{12}$. As limitações do rastreamento incluem pior acesso principalmente entre mulheres de baixa renda; a dificuldade de seguimento das mulheres em um sistema de registro centralizado no exame; e a captação das mulheres realizada de forma não organizada e que não segue a recomendação de periodicidade ${ }^{22}$.

As falhas apontadas impulsionaram a busca por técnicas alternativas (associadas ou substitutas) que viessem contribuir com a redução das perdas e identificação mais precisa de mulheres portadoras dos tipos de HPV com maior risco oncogênico. As técnicas de biologia molecular vêm contribuindo para o conhecimento da infecção genital pelo HPV em diferentes cenários, sendo utilizadas em pesquisas científicas desde o início dos anos 1980, mas só mais recentemente foram incorporadas aos serviços de saúde ${ }^{5,30,31}$.

No Brasil, a identificação de mulheres infectadas pelo HPV é feita a partir de estudos heterogêneos e, por isso, de comparabilidade difícil e reprodutibilidade limitada, pois estimativas elaboradas a partir de estudos que consideram mulheres referenciadas podem resultar em medidas superestimadas ${ }^{2}$. As estimativas de prevalência e os fatores associados à infecção por HPV permitem compreender por que determinados grupos de mulheres encontram-se mais vulneráveis que outros de modo a possibilitar propostas de ações de prevenção específicas para tais grupos que aumentem a efetividade da prevenção primária e secundária do câncer do colo do útero, que é uma doença evitável ${ }^{14}$.

Dessa forma, o objetivo do presente estudo foi estimar a prevalência e os fatores de risco para infecção pelo HPV entre mulheres residentes em área de cobertura da Estratégia Saúde da Família (ESF).

\section{MÉTODOS}

Foi realizado um estudo transversal com população de residentes em área adscrita a duas unidades da Estratégia Saúde da Família, localizadas na periferia do município de Juiz de Fora, MG. Foram consideradas elegíveis todas as mulheres residentes em área de cobertura da ESF com idade entre 20 e 59 anos, assintomáticas. Foram excluídas mulheres grávidas, aquelas que se apresentavam imunodeprimidas ou que foram submetidas previamente a procedimentos de excisão do útero (histerectomia, conização). Aproximadamente 3.500 mulheres foram recrutadas em seus domicílios pelos agentes comunitários de saúde para submeterem-se ao exame preventivo na área proposta, enquanto ação programática 
desenvolvida pelas unidades de saúde da família, com ênfase no rastreamento organizado das faltosas através do cadastro das ESF.

Os dados foram coletados no período de setembro de 2010 a agosto de 2012. As participantes do estudo foram entrevistadas por profissionais de saúde treinados, utilizando questionário padronizado adaptado da Pesquisa Nacional de Saúde, aplicado no Distrito Federal em 2010, aprimorado a partir de 2013 por meio da parceria entre a Fundação Oswaldo Cruz e o Instituto Brasileiro de Geografia e Estatística. O instrumento final englobou grupos de questões distribuídas por nove módulos: identificação, características sociodemográficas, apoio social, autoavaliação do estado de saúde, estilo de vida, morbidade, saúde da mulher, comportamento sexual e infecções sexualmente transmissíveis, dados aferidos. O instrumento, seu detalhamento e a definição de cada categoria utilizada encontra-se disponível para consulta. Foram aferidos dados antropométricos, dados de morbidade (pressão arterial) e os resultados de exames referem-se a laudos trazidos pelas participantes ${ }^{6}$.

A seguir, as mulheres foram submetidas ao exame citológico cervical convencional nas unidades básicas de saúde, realizando simultaneamente teste para infecção pelo HPV, sendo as amostras coletadas por enfermeiros e médicos treinados atuantes nas unidades.

A testagem para HPV foi realizada no Laboratório de Virologia do Instituto de Medicina Tropical da Universidade de São Paulo. As amostras do colo uterino foram preservadas em solução PreservCyt e analisadas pelo método de Polymerase Chain Reaction (PCR) utilizando o teste HPV-HR test + GT 16/18 na plataforma automatizada cobas 4800 HPV Test ${ }^{\circledR}$ (Roche Molecular Systems, Inc., Branchburg, New Jersey, USA), o qual utiliza iniciadores ("primers") para amplificar o DNA de 14 tipos de HPV de alto risco (HPV-AR): HPV16, HPV18 e um "pooled primer" com os HR-HPV 31, 33, 35, 39, 45, 51, 52, 56, 58, 59, 66 e 68. Foram considerados resultados negativos os espécimes em que não houve amplificação do DNA viral e positivos aqueles em que houve amplificação do DNA viral correspondente aos primers do teste. Os procedimentos de análise da qualidade das amostras ocorreram conforme protocolos pré-estabelecidos, adotados pelo Laboratório executor.

No período estudado, 2.076 mulheres elegíveis, aproximadamente $60 \%$ da população de mulheres residentes nas áreas adscritas pela ESF, atenderam ao recrutamento, compareceram às unidades de ESF e foram incluídas no estudo. Devido a problemas na leitura, não foi possível obter resultado do teste de HPV para 54 mulheres (2,6\%), pois 40 amostras tiveram resultados inválidos e 14, inconclusivos. Assim, a população de estudo contou com 2.022 mulheres. Em uma das unidades de saúde, o número de mulheres estudado foi quase o dobro do obtido na outra unidade mantendo a proporcionalidade do tamanho das populações adstritas a essas duas unidades.

Procedeu-se a análise univariada dos dados, sendo calculadas as frequências absoluta e relativa para as variáveis categóricas nominais, segundo unidade de saúde da família. Em seguida, foi realizada a análise bivariada paralelamente à comparação das características básicas entre os grupos, utilizando-se o teste Qui-quadrado para proporções, considerado significativo se $p \leq 0,20$. Foram estimadas as medidas de prevalência de infecção pelo HPV, geral e estratificadas segundo variáveis selecionadas com respectivos intervalos com 95\% de confiança de (IC95\%) e valor de p.

Para avaliar os fatores relacionados à infecção, identificada como o resultado positivo para o teste de HPV, foram selecionadas variáveis atendendo a critérios epidemiológicos e de plausibilidade biológica. Foram incluídas no estudo características relativas a: faixa etária, escolaridade, cor da pele, renda familiar per capita, avaliação de saúde, estilo de vida (consumo de álcool, tabagismo atual e prévio), história reprodutiva (idade da menarca, uso de métodos contraceptivos, realização de exame preventivo, paridade) e comportamento sexual (idade da sexarca, número de parceiros sexuais ao longo da vida, histórico de infecções sexualmente transmissíveis). As categorias das variáveis foram definidas conforme referências clássicas da literatura, selecionadas no desenvolvimento do questionário. A variável renda per capita foi categorizada tomando-se por base os valores de salário mínimo à época do estudo (R \$603,31, o qual foi aproximado para $\mathrm{R} \$ 600,00)$. Desta forma, renda baixa correspondeu a valores 
abaixo de 50\% do salário mínimo, renda média correspondeu a valores acima de $50 \%$ até um salário mínimo e renda alta mais de um salário mínimo. Foi considerado o rendimento total da família, incluindo todos os moradores do domicílio.

Foram calculadas razões de prevalência brutas e ajustadas por meio de regressão de Poisson com variância robusta e os respectivos intervalos com $95 \%$ de confiança. As variáveis que na análise bivariada tiveram $\mathrm{p} \leq 0,20$ foram selecionadas para o modelo final, o qual, além dessas, incluiu a faixa etária e unidade da saúde da família na qual a mulher residia e foi atendida. Todas as análises foram realizadas utilizando o programa estatístico Stata, versão 12 (Data Analysis and Statistic Software, StataCorp LP, College Station, Texas, USA).

O projeto foi aprovado pela Comissão de Ética do Instituto de Medicina Social da Universidade do Estado do Rio de Janeiro (Parecer 0026.1.259.180-09), seguindo todas as recomendações propostas. Todas as mulheres incluídas no estudo o fizeram mediante leitura e assinatura do termo de consentimento esclarecido e informado, ficando assegurada a confidencialidade dos dados e a privacidade das mulheres participantes em todas as etapas do estudo.

\section{RESULTADOS}

As características sociodemográficas, bem como aquelas relativas à autoavaliação de saúde, estilo de vida, saúde reprodutiva e comportamento sexual das mulheres estudadas encontram-se descritas na Tabela 1.

A prevalência global de infecção por pelo menos um tipo de HPV de alto risco entre mulheres das duas unidades de ESF foi igual a 12,6\% (IC95\% 11,16-14,05). A prevalência de infecção por pelo menos um tipo de HPV-AR, exceto HPV16 e HPV18 (pooled primer com 12 tipos) foi igual a 8,6\% (IC95\% 7,30-9,77), enquanto a prevalência de infecção por HPV 16 e 18 foi igual a 1,8\% (IC95\% 1,20-2,35) e 0,5\% (IC95\% 0,22-0,86), respectivamente. A prevalência de coinfecção por HPV16 ou HPV18 e pelo menos um dos 12 tipos do pooled primer (31, 33, 35, $39,45,51,52,56,58,59,66$ e 68) foi igual a 1,7\% (IC95\% 1,16-2,29) (Figura).

A prevalência de infecção pelo HPV segundo variáveis independentes selecionadas é apresentada na Tabela 2. Na análise bivariada, as seguintes variáveis mostraram-se associadas a maiores prevalências de infecção por HPV: estado conjugal (solteira: $\mathrm{RP}=2,26$; IC95\% 1,79-2,84), faixa etária (20-24 anos: $\mathrm{RP}=5,34$; IC95\% 3,65-7,79), escolaridade (ensino fundamental completo:

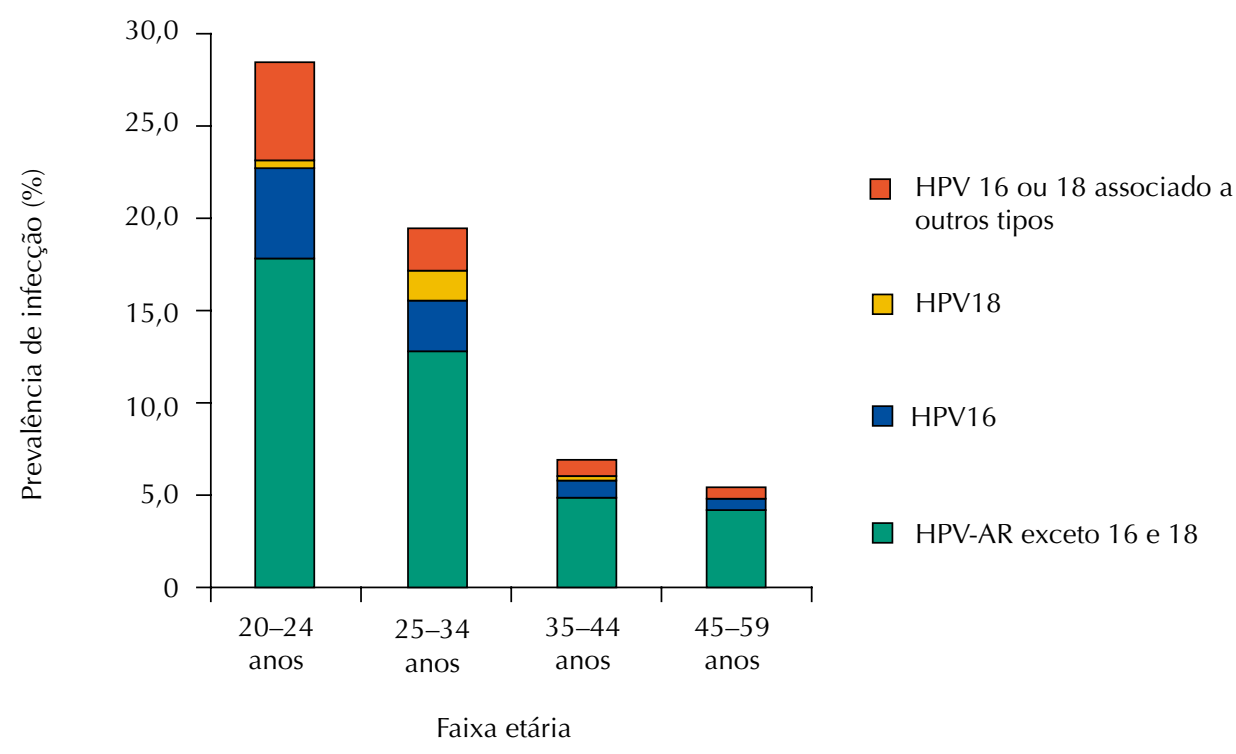

Figura. Prevalência de infecção por tipos de HPV segundo faixa etária entre mulheres estudadas. Juiz de Fora, MG. 
Tabela 1. Características sociodemográficas, condições de habitação, autoavaliação de saúde, estilo de vida, saúde reprodutiva e comportamento sexual entre mulheres residentes em área de cobertura da Estratégia Saúde da Família. Juiz de Fora, MG, 2010-2012.

\begin{tabular}{|c|c|c|c|c|c|c|c|}
\hline \multirow{2}{*}{ Variável } & \multirow{2}{*}{ Categoria } & \multicolumn{2}{|c|}{ Unidade I } & \multicolumn{2}{|c|}{ Unidade II } & \multicolumn{2}{|c|}{ Total } \\
\hline & & $\mathbf{n}$ & $\%$ & $\mathbf{n}$ & $\%$ & $\mathrm{n}^{\mathrm{a}}$ & $\%$ \\
\hline \multirow[t]{8}{*}{ Faixa etária (anos) } & $20-24$ & 151 & 11,6 & 107 & 13,9 & 258 & 12,5 \\
\hline & $25-29$ & 181 & 14,0 & 98 & 12,8 & 279 & 13,5 \\
\hline & $30-34$ & 179 & 13,8 & 124 & 16,2 & 303 & 14,7 \\
\hline & $35-39$ & 175 & 13,5 & 105 & 13,7 & 280 & 13,6 \\
\hline & $40-44$ & 167 & 12,9 & 107 & 13,9 & 274 & 13,3 \\
\hline & $45-49$ & 157 & 12,1 & 89 & 11,6 & 246 & 11,9 \\
\hline & $50-54$ & 149 & 11,5 & 73 & 9,5 & 222 & 10,8 \\
\hline & $55-59$ & 136 & 10,5 & 64 & 8,3 & 200 & 9,7 \\
\hline \multirow[t]{2}{*}{ Estado conjugal } & Solteira & 296 & 22,9 & 132 & 17,2 & 428 & 20,8 \\
\hline & Com companheiro & 998 & 77,1 & 635 & 82,8 & 1.633 & 79,2 \\
\hline \multirow[t]{5}{*}{ Anos de estudo } & $<1$ & 8 & 0,6 & 13 & 1,7 & 21 & 1,0 \\
\hline & $1-3$ & 89 & 6,9 & 50 & 6,6 & 139 & 6,8 \\
\hline & $4-7$ & 474 & 36,9 & 298 & 39,4 & 772 & 37,8 \\
\hline & $8-10$ & 257 & 20,0 & 167 & 22,1 & 424 & 20,8 \\
\hline & $>11$ & 456 & 35,5 & 228 & 30,1 & 684 & 33,5 \\
\hline \multirow[t]{2}{*}{ Cor da pele } & Branca & 628 & 48,5 & 342 & 44,6 & 970 & 47,0 \\
\hline & Não branca & 667 & 51,5 & 425 & 55,4 & 1.092 & 53,0 \\
\hline \multirow[t]{2}{*}{ Pratica religião } & Sim & 1.260 & 99,1 & 728 & 96,8 & 1.988 & 98,3 \\
\hline & Não & 11 & 0,9 & 24 & 3,2 & 35 & 1,7 \\
\hline \multirow[t]{3}{*}{ Renda per capita } & Baixa & 478 & 36,9 & 299 & 39,0 & 777 & 37,7 \\
\hline & Média & 771 & 59,5 & 443 & 57,8 & 1.214 & 28,9 \\
\hline & Alta & 46 & 3,5 & 25 & 3,3 & 71 & 7,4 \\
\hline \multirow[t]{2}{*}{ Água canalizada } & Rede geral & 1.283 & 99,5 & 748 & 97,5 & 2.031 & 98,7 \\
\hline & Outras & 7 & 0,5 & 19 & 2,5 & 26 & 1,3 \\
\hline \multirow{2}{*}{ Destino do lixo } & Coleta regular & 1.286 & 99,5 & 753 & 98,2 & 2.039 & 99,0 \\
\hline & Outros & 7 & 0,54 & 14 & 1,8 & 21 & 1,0 \\
\hline \multirow[t]{2}{*}{ Escoadouro } & Rede de esgoto & 1.266 & 97,8 & 700 & 91,3 & 1.966 & 95,3 \\
\hline & Outros & 29 & 2,2 & 67 & 8,7 & 96 & 4,7 \\
\hline \multirow[t]{2}{*}{ Avaliação de saúde } & Ruim a regular & 525 & 40,7 & 336 & 43,8 & 861 & 41,9 \\
\hline & Boa a muito boa & 764 & 59,3 & 431 & 56,2 & 1.195 & 58,1 \\
\hline \multirow[t]{2}{*}{ Consumo de álcool } & Não & 698 & 53,9 & 481 & 62,7 & 1.179 & 57,2 \\
\hline & Sim & 597 & 46,1 & 286 & 37,3 & 883 & 42,8 \\
\hline \multirow[t]{2}{*}{ Tabagismo atual } & Não & 986 & 77,2 & 616 & 80,4 & 1.602 & 78,4 \\
\hline & Sim & 291 & 22,8 & 150 & 19,6 & 441 & 21,6 \\
\hline \multirow[t]{2}{*}{ Ex-fumante } & Não & 728 & 71,0 & 459 & 72,6 & 1.187 & 71,6 \\
\hline & Sim & 298 & 29,0 & 173 & 27,4 & 471 & 28,7 \\
\hline Idade da menarca & $\leq 12$ anos & 589 & 46,2 & 316 & 42,1 & 905 & 44,6 \\
\hline & $\leq 13$ anos & 687 & 53,8 & 435 & 57,9 & 1.122 & 55,3 \\
\hline Exame & Há 3 ou - anos & 1.016 & 78,5 & 585 & 76,3 & 1.601 & 77,7 \\
\hline Papanicolaou & Atraso ${ }^{b} /$ Nunca fez & 278 & 21,5 & 182 & 23,7 & 460 & 22,3 \\
\hline Contracepção & Qualquer método & 872 & 67,6 & 536 & 69,9 & 1.408 & 68,4 \\
\hline & Nenhum método & 418 & 32,4 & 231 & 30,1 & 649 & 31,4 \\
\hline Nuliparidade & Não & 1.134 & 87,6 & 669 & 87,3 & 1.803 & 87,5 \\
\hline & Sim & 161 & 12,4 & 97 & 12,7 & 258 & 12,5 \\
\hline Idade da sexarca & $\geq 16$ anos & 278 & 21,6 & 170 & 22,7 & 448 & 22,0 \\
\hline & $\leq 15$ anos & 1.008 & 78,4 & 580 & 77,3 & 1.588 & 78,0 \\
\hline Parceiros sexuais ${ }^{c}$ & Até 3 & 903 & 73,1 & 530 & 72,3 & 1.433 & 72,8 \\
\hline & Mais de 3 & 333 & 26,9 & 203 & 27,7 & 536 & 27,2 \\
\hline Teste para sífilis & Negativo & 635 & 98,3 & 315 & 98,4 & 950 & 98,3 \\
\hline & Positivo & 11 & 1,7 & 5 & 1,6 & 16 & 1,7 \\
\hline Teste para HIV & Negativo & 825 & 99,6 & 456 & 98,9 & 1.281 & 99,4 \\
\hline & Positivo & 3 & 0,4 & 5 & 1,1 & 8 & 0,6 \\
\hline
\end{tabular}

${ }^{a}$ Total de mulheres com resultado de testagem de HPV e com informação válida sobre a variável.

${ }^{\mathrm{b}}$ Exame citológico cervical realizado há mais de três anos.

c Ao longo da vida. 
$\mathrm{RP}=2,26$; IC95\% 1,79-2,84), avaliação de saúde (boa a muito boa: $\mathrm{RP}=1,40$; IC95\% 1,10-1,79), consumo de álcool (qualquer frequência: $\mathrm{RP}=1,89$, IC95\% 1,49-2,38) tabagismo (atual: $\mathrm{RP}=1,36$, IC95\% 1,05-1,76), uso de método contraceptivo (qualquer método: $\mathrm{RP}=1,68$; IC95\% $1,26-2,23)$, nuliparidade $(\mathrm{RP}=2,04$; IC95\% 1,57-2,66), sexarca com idade $\leq 15$ anos $(\mathrm{RP}=1,49$; IC95\% 1,17-1,92) e parceiros sexuais ao longo da vida (número $\geq 3$ : $R P=1,84$; IC95\% 1,45-2,33).

$\mathrm{Na}$ análise multivariada permaneceram significativamente associadas à infecção pelo HPV as variáveis ser solteira $(\mathrm{RP}=1,40 ; \mathrm{IC} 95 \% 1,07-1,84)$, ter consumido álcool com qualquer frequência $(\mathrm{RP}=1,44$; IC95\% 1,11-1,86) e ter tido três ou mais parceiros sexuais ao longo da vida (RP = 1,35; IC95\% 1,04-1,74) (Tabela 3).

Tabela 2. Prevalência e razão de prevalência bruta de infecção pelo HPV segundo variáveis selecionadas entre mulheres residentes em área de cobertura da Estratégia Saúde da Família. Juiz de Fora, MG, 2010-2012.

\begin{tabular}{|c|c|c|c|c|c|c|c|c|}
\hline \multirow{2}{*}{ Variável } & \multirow{2}{*}{ Categorias } & \multirow{2}{*}{ Total* } & \multicolumn{3}{|c|}{ HPV+ } & \multirow{2}{*}{ RP* bruta } & \multirow{2}{*}{ IC95\% } & \multirow{2}{*}{$p$} \\
\hline & & & $\mathbf{n}$ & $\%$ & IC95\% & & & \\
\hline \multirow[t]{5}{*}{ Faixa etária (anos) } & $20-24$ & 246 & 70 & 28,4 & $22,90-34,53$ & 5,34 & $3,65-7,79$ & $<0,001$ \\
\hline & $25-34$ & 575 & 112 & 19,5 & $16,31-22,95$ & 3,65 & $2,54-5,25$ & $<0,001$ \\
\hline & $35-44$ & 544 & 38 & 7,0 & $4,99-9,46$ & 1,31 & $0,84-2,03$ & 0,233 \\
\hline & $45-59$ & 657 & 35 & 5,3 & $3,73-7,33$ & 1 & - & - \\
\hline & Total & 2.022 & 255 & 12,6 & $11,19-14,13$ & & & \\
\hline \multirow[t]{2}{*}{ Estado conjugal } & Com companheiro & 1.601 & 160 & 10,0 & $8,56-11,56$ & 1 & - & - \\
\hline & Solteira & 420 & 95 & 22,6 & $18,70-26,92$ & 2,26 & $1,79-2,84$ & $<0,001$ \\
\hline \multirow[t]{2}{*}{ Ensino fundamental } & Incompleto & 914 & 73 & 8,0 & $6,31-9,93$ & 1 & - & - \\
\hline & Completo & 1.086 & 179 & 16,5 & $14,32-18,82$ & 2,06 & $1,59-2,67$ & $<0,001$ \\
\hline \multirow[t]{2}{*}{ Cor da pele } & Branca & 951 & 115 & 12,1 & $10,08-14,33$ & 1 & - & - \\
\hline & Não branca & 1.071 & 140 & 13,17 & $11,10-15,23$ & 1,08 & $0,85-1,36$ & 0,508 \\
\hline \multirow[t]{2}{*}{ Baixa renda familiar } & Não & 1.263 & 155 & 12,3 & $10,51-14,20$ & 1 & - & - \\
\hline & Sim & 759 & 100 & 13,2 & $10,84-15,79$ & 1,07 & $0,84-1,35$ & 0,553 \\
\hline \multirow[t]{2}{*}{ Avaliação de saúde } & Muito ruim a regular & 842 & 86 & 10,2 & $8,55-12,45$ & 1 & - & - \\
\hline & Boa a muito boa & 1.174 & 169 & 14,4 & $12,53-16,43$ & 1,40 & $1,10-1,79$ & 0,006 \\
\hline \multirow[t]{2}{*}{ Consumo de álcool } & Não (nunca) & 1.152 & 105 & 9,1 & $7,51-10,92$ & 1 & - & - \\
\hline & Sim & 870 & 150 & 17,2 & $14,78-19,91$ & 1,89 & $1,49-2,38$ & $<0,001$ \\
\hline \multirow[t]{2}{*}{ Tabagismo atual } & Não & 1.569 & 183 & 11,7 & $10,11-13,35$ & 1 & - & - \\
\hline & Sim & 434 & 69 & 15,9 & $12,58-19,68$ & 1,36 & $1,05-1,76$ & 0,018 \\
\hline \multirow[t]{2}{*}{ Ex-fumante } & Sim & 465 & 50 & 10,7 & $8,08-13,92$ & 1 & - & - \\
\hline & Não & 1.160 & 142 & 12,2 & $10,40-14,26$ & 1,13 & $0,84-1,54$ & 0,403 \\
\hline \multirow[t]{2}{*}{ Menarca $\leq 12$ anos } & Sim & 888 & 107 & 12,0 & $9,98-14,37$ & 1 & - & - \\
\hline & Não & 1.099 & 142 & 12,9 & $10,99-15,04$ & 1,07 & $0,84-1,35$ & 0,560 \\
\hline \multirow[t]{2}{*}{$\begin{array}{l}\text { Exame Papanicolaou em } \\
\text { atraso }\end{array}$} & Não & 1.574 & 202 & 12,8 & $11,21-14,58$ & 1 & - & - \\
\hline & Sim & 447 & 53 & 11,8 & $9,00-15,22$ & 0,92 & $0,69-1,22$ & 0,585 \\
\hline \multirow[t]{2}{*}{ Usa contraceptivos } & Não & 638 & 55 & 8,6 & $6,56-11,07$ & 1 & - & - \\
\hline & Sim & 1.379 & 200 & 14,5 & $12,68-16,47$ & 1,68 & $1,26-2,23$ & $<0,001$ \\
\hline \multirow[t]{2}{*}{ Gravidez prévia } & Sim & 1.772 & 198 & 11,2 & $9,74-12,73$ & 1 & - & - \\
\hline & Não & 249 & 57 & 22,9 & $17,82-28,61$ & 2,04 & $1,57-2,66$ & $<0,001$ \\
\hline \multirow[t]{2}{*}{ Sexarca $\leq 15$ anos } & Não & 1.559 & 178 & 11,4 & $9,88-13,10$ & 1 & - & - \\
\hline & Sim & 438 & 75 & 17,1 & $13,71-20,98$ & 1,49 & $1,17-1,92$ & 0,001 \\
\hline \multirow[t]{2}{*}{ Até 3 parceiros sexuais } & Sim & 1.400 & 142 & 10,1 & $8,61-11,84$ & 1 & - & - \\
\hline & Não & 530 & 99 & 18,7 & $15,44-22,26$ & 1,84 & $1,45-2,33$ & $<0,001$ \\
\hline \multirow[t]{2}{*}{ Teste - sífilis positivo } & Não & 935 & 138 & 14,7 & $12,54-17,19$ & 1 & - & - \\
\hline & Sim & 16 & 1 & 6,2 & $1,58-30,23$ & 0,42 & $0,63-2,84$ & 0,377 \\
\hline \multirow[t]{2}{*}{ Teste - HIV positivo } & Não & 175 & 1.258 & 13,9 & $12,04-15,94$ & 1 & - & - \\
\hline & Sim & 1 & 8 & 12,5 & $3,15-52,65$ & 0,89 & $0,14-5,65$ & 0,909 \\
\hline
\end{tabular}

\footnotetext{
* Total de mulheres com resultado de testagem de HPV e com informação válida sobre a variável.
} 
Tabela 3. Razões brutas e ajustadas de prevalência de infecção pelo HPV para as variáveis selecionadas entre mulheres residentes em área de cobertura da Estratégia Saúde da Família. Juiz de Fora, MG, 2010-2012.

\begin{tabular}{|c|c|c|c|c|c|c|}
\hline Variável & Categoria & RP bruta & IC95\% & p & RP ajustada & IC95\% \\
\hline \multirow[t]{4}{*}{ Faixa etária (anos) } & $45-59$ & 1 & - & - & 1 & - \\
\hline & $35-44$ & 1,31 & $0,84-2,03$ & 0,233 & 1,08 & $0,67-1,74$ \\
\hline & $25-34$ & 3,65 & $2,54-5,25$ & $<0,001$ & 2,66 & $1,74-4,07$ \\
\hline & $20-24$ & 5,34 & $3,65-7,79$ & $<0,001$ & 3,59 & $2,23-5,78$ \\
\hline \multirow[t]{2}{*}{ Estado conjugal } & Casada (lifetime) & 1 & - & - & 1 & - \\
\hline & Solteira (lifetime) & 2,26 & $1,79-2,84$ & $<0,001$ & 1,40 & $1,07-1,84$ \\
\hline \multirow[t]{2}{*}{ Ensino fundamental } & Incompleto & 1 & - & - & 1 & - \\
\hline & Completo & 2,06 & $1,59-2,67$ & $<0,001$ & 1,11 & $0,82-1,50$ \\
\hline \multirow[t]{2}{*}{ Avaliação de saúde } & Muito ruim a regular & 1 & - & - & 1 & - \\
\hline & Boa a muito boa & 1,40 & $1,10-1,79$ & 0,006 & 1,15 & $0,90-1,48$ \\
\hline \multirow[t]{2}{*}{ Consumo de álcool } & Não (nunca) & 1 & - & - & 1 & - \\
\hline & Sim & 1,89 & $1,49-2,38$ & $<0,001$ & 1,44 & $1,11-1,86$ \\
\hline \multirow[t]{2}{*}{ Tabagismo atual } & Não & 1 & - & - & 1 & - \\
\hline & Sim & 1,36 & $1,05-1,76$ & 0,018 & 1,23 & $0,92-1,64$ \\
\hline \multirow[t]{2}{*}{ Contracepção } & Nenhum método & 1 & - & - & 1 & - \\
\hline & Qualquer método & 1,68 & $1,26-2,23$ & $<0,001$ & 1,01 & $0,75-1,36$ \\
\hline \multirow[t]{2}{*}{ Nuliparidade } & Não & 1 & - & - & 1 & - \\
\hline & Sim & 2,04 & $1,57-2,66$ & $<0,001$ & 1,11 & $0,80-1,55$ \\
\hline \multirow[t]{2}{*}{ Idade da sexarca } & $\geq 16$ anos & 1 & - & - & 1 & - \\
\hline & $\leq 15$ anos & 1,49 & $1,17-1,92$ & 0,001 & 0,95 & $0,72-1,26$ \\
\hline \multirow[t]{2}{*}{$\mathrm{N}^{\circ}$ de parceiros sexuais } & Até 3 (lifetime) & 1 & - & - & 1 & - \\
\hline & Mais de 3 (lifetime) & 1,84 & $1,45-2,33$ & $<0,001$ & 1,35 & $1,04-1,74$ \\
\hline
\end{tabular}

\section{DISCUSSÃO}

A prevalência de infecção do colo do útero pelo HPV em mulheres atendidas pela Estratégia Saúde da Família foi 12,6\%. Essa medida aproxima-se da prevalência (12,8\%) observada por Girianelli et al. (2010) em estudo com recrutamento domiciliar de mulheres de baixa renda residentes em municípios da Baixada Fluminense, RJ, que utilizou a técnica de captura híbrida para detecção de HPV. Embora a técnica de PCR venha a apresentar estimativas de prevalências mais altas que as obtidas com emprego da técnica de captura híbrida, a obtenção da medida no estudo de Girianelli et al. (2010) resultou da inclusão de mulheres que estavam há mais de três anos sem fazer o exame preventivo, enquanto o presente estudo considerou dados de todas as mulheres da população alvo para o rastreamento ${ }^{11,13}$.

O comparecimento das mulheres da população-alvo para o rastreamento do câncer do colo do útero às unidades de ESF participantes no presente estudo foi aquém do esperado (60\%). Esperava-se que, por ser uma ação iniciada com recrutamento nos domicílios e agendamento de consultas na unidade de saúde, a procura das mulheres fosse mais alta. A adesão reduzida ao rastreamento reflete o comportamento observado em outros estudos brasileiros e pode ter influenciado as estimativas observadas para o grupo como um todo. Isso mostra que, mesmo no rastreamento organizado, parece haver fatores que influenciam na resposta ao recrutamento, resultando em adesão parcial ${ }^{15}$. Deve-se considerar que parte das mulheres que não vieram à unidade podem ter realizado o exame preventivo em serviços fora do SUS, por terem plano de saúde ou mesmo por terem pagado pelo exame em serviço privado. Essa limitação, entretanto, não necessariamente compromete a representatividade dos achados da área geográfica uma vez que parece haver pouca variação no status socioeconômico entre as mulheres residentes.

Com relação às perdas na testagem do HPV, apesar do treinamento e supervisão periódicos, houve falhas na captação de resultados de tipagem e detecção de HPV e de citologia, sem 
impacto significativo na análise dos resultados. Tais perdas eram de certo modo previstas, considerando que o estudo foi operacionalizado via serviço de saúde.

Os resultados relativos aos tipos de HPV circulantes do presente estudo mostraram-se discordantes de alguns estudos que avaliaram a prevalência de HPV entre mulheres brasileiras ${ }^{16}$. A prevalência da infecção pelos tipos 16 e 18 se assemelha às prevalências mundiais, sendo a infecção pelo HPV16 a mais frequente, seja isolada ou em coinfecções, seguida pela infecção isolada por HPV18 isoladamente, sugerindo menor prevalência que os tipos contidos no pooled primer HPV de alto risco 4 . Não foi possível identificar quais tipos os sucederiam virtualmente no nicho ecológico, pois o pooled primer utilizado incluiu apenas 12 tipos de HPV de alto risco. Contudo, a prevalência de infecção pelos tipos contidos neste primer foi aproximadamente cinco vezes maior que a prevalência de infecção isolada pelo HPV16. Assumindo uma hipotética distribuição homogênea entre os tipos do primer, esta prevalência pode ser interpretada em caráter preliminar como prevalência de $0,71 \%$ para cada tipo, precedendo a infecção isolada pelo HPV18 em magnitude e relevância. Como essa distribuição homogênea seguramente não ocorre, a diferença das prevalências entre determinados tipos de HPV de alto risco e o HPV18 pode ser ainda maior.

A prevalência de infecção do colo do útero pelo HPV varia de média a alta, principalmente entre as mulheres jovens, que já iniciam atividade sexual com risco de exposição aos tipos oncogênicos. A incorporação da testagem de HPV nas mulheres rastreadas traria como vantagem o aumento do intervalo de seguimento, com as mulheres HPV-positivas devendo realizar exame citológico, enquanto as mulheres HPV-negativas só precisariam de intervalos de cinco anos, dois anos a mais que o preconizado para o seguimento com exame citológico após dois anos com resultados normais ${ }^{5,20}$.

A prevalência de infecção pelo HPV em estudos brasileiros realizados com mulheres recrutadas em unidades de saúde parece ser consideravelmente maior que a prevalência observada nos estudos de base populacional. Nesses últimos, as possibilidades de viés de seleção e consequente superestimação das estimativas de prevalência são praticamente eliminadas, uma vez a população estudada não inclui somente mulheres que foram atendidas ou referenciadas a serviços de saúde por serem sintomáticas.

Quanto aos fatores relacionados à infecção do colo do útero pelo HPV observados, o estado conjugal (solteira), o consumo de álcool (qualquer frequência) e o número de parceiros sexuais ao longo da vida (três ou mais) permaneceram como fatores preditivos independentes da infecção por HPV, mesmo após ajustes por outras variáveis. Estes achados ratificam os resultados de outros estudos que também relacionaram o risco de infecção pelo HPV ao estado conjugal, ao consumo do álcool abusivo e à história sexual das mulheres 1,3,7,8,11,18,23-25,27. Não se investigou a associação entre ter relacionamentos extraconjugais ou ter parceiros com relacionamentos extraconjugais e a prevalência de infecção pelo HPV, fator que se mostrou associado à prevalência de infecção em alguns estudos.

A autoavaliação do estado de saúde, o tabagismo, a utilização de métodos contraceptivos, a paridade e a idade da sexarca não permaneceram como preditivos independentes da infecção por HPV no modelo final. Vaccarella et al. (2006) também não encontraram associação estatisticamente significativa entre a infecção por HPV e paridade, uso prolongado de contraceptivos orais ou uso de preservativos pelo parceiro sexual e sexarca ${ }^{26,27}$. O tabagismo tem sido apontado como fator associado à persistência e ao aparecimento da neoplasia mas não ao risco de infecção, tendo sido descartada a sua associação com a infecção pelo HPV²8.

Cabe destacar que a prevalência da infecção nas diferentes faixas etárias é próxima à estimada em estudos similares, sendo a maior proporção de infectadas encontrada entre mulheres de 25 a 34 anos, que também é a faixa etária com maior incidência de carcinoma in situ $u^{17,18,25}$.

Assim, a prevalência de infecção entre as mulheres estudadas é semelhante àquela encontrada em outros estudos brasileiros, sendo mais elevada entre mulheres mais jovens. Os fatores preditivos independentes de infecção por HPV - situação conjugal, consumo de álcool e 
número de parceiros sexuais durante a vida - são altamente influenciados por questões econômicas, culturais e sociais. Estudos adicionais são necessários para compreender se a relação entre os fatores associados a maior prevalência de infecção pelo HPV se modifica quando analisada frente à incidência de lesões pré-invasivas e invasivas do câncer do colo do útero entre estas mulheres, para verificar se os fatores preditivos seriam os mesmos em uma população de mulheres com carcinoma (in situ ou invasor).

O rastreamento organizado, alternativo ao oportunístico, prevê a captação de mulheres nas faixas etárias alvo, ao mesmo tempo em que não repete desnecessariamente o Papanicolaou. No entanto, fatores relacionados a não adesão ao exame dentro da periodicidade recomendada precisam ser reconhecidos e tornar-se alvo de intervenção, pois a simples mudança na estratégia de captação não é garantia para que a mulher compareça à unidade de saúde para ser examinada. A testagem para o HPV, bem como o exame preventivo, teria melhor desempenho num sistema de rastreamento organizado, não oportunístico.

A testagem para o HPV deve ser avaliada economicamente para que sua implantação seja recomendada. Enquanto tecnologia a ser incorporada no rastreamento, requer treinamento das equipes e supervisão logística rigorosa para evitar contaminação de amostras negativas ou contaminação cruzada por tipos entre amostras. Faz-se necessário que sejam adotados métodos baseados em reação de cadeia de polimerase que reconheçam cada tipo de HPV na prevalência de infecção entre as mulheres das diversas regiões do país. A maior sensibilidade e menor especificidade do teste de HPV-DNA comparado ao rastreamento com citologia convencional poderiam sobrecarregar as referências de atenção secundária. O impacto desse aumento de positividade sobre os serviços precisa ser avaliado, inclusive na sua relação de custo-efetividade. Por outro lado, uma modificação na faixa etária de mulheres rastreadas e a utilização do exame em regiões de acesso restrito às unidades de saúde beneficiariam mulheres que vivem em desigualdade de acesso à prevenção do câncer do colo do útero, identificando mulheres em maior risco para aquisição e persistência da infecção e, consequentemente, para evolução das lesões preneoplásicas. As implicações éticas da testagem de HPV precisam ser consideradas, e o impacto psicológico avaliado criteriosamente, uma vez que as mulheres que apresentam infecção pelo HPV não estão doentes, e pode haver dificuldade na compreensão desta condição. A testagem fornece um panorama da prevalência de infecção pelo HPV em momento de necessidade de vigilância de tipos circulantes, inclusive preenchimento de nicho ecológico, subsequente à implantação de vacina contra os tipos 16 e 18 e esta ação pode ser encarada como um esforço dentro das prioridades das políticas voltadas para o controle do câncer do colo do útero e, portanto, para a saúde da mulher.

De um modo geral, ações que enfoquem os determinantes sociais da saúde, na abordagem do curso, qualidade e estilo de vida, promovem melhorias essenciais à modificação da exposição aos fatores de risco relacionados à infecção pelo HPV, bem como para muitas outras exposições, doenças e agravos. As questões relacionadas ao gênero e suas vulnerabilidades devem pautar o planejamento das ações educativas junto às mulheres, permeando as ações de prevenção e controle de agravos antes mesmo da atividade sexual se iniciar. O setor saúde deve trabalhar de forma integrada com outros setores para garantir a superação das desigualdades de acesso aos serviços e às informações qualificadas sobre a saúde da mulher, identificando as oportunidades que lhe cabem para que esta integração se dê. A busca de resolutividade para esses problemas não será completa sem que a pesquisa epidemiológica e o serviço de saúde dialoguem, num processo de benefícios mútuos, onde a maior favorecida certamente será a população feminina.

\section{REFERÊNCIAS}

1. Alibegashvili T, Clifford GM, Vaccarella S, Baidoshvili A, Gogiashvili L, Tsagareli Z, et al. Human papillomavirus infection in women with and without cervical cancer in Tbilisi, Georgia. Cancer Epidemiol. 2011;35(5):465-70. https://doi.org/10.1016/j.canep.2010.12.006 
2. Ayres ARG, Azevedo e Silva G. Prevalência de infecção do colo do útero pelo HPV no Brasil: revisão sistemática. Rev Saude Publica. 2010;44(5):963-74. https://doi.org/10.1590/S0034-89102010000500023

3. Bardin A, Vaccarella S, Clifford GM, Lissowska J, Rekosz M, Bobkiewicz P, et al. Human papillomavirus in women with and without cervical cancer in Warsaw, Poland. Eur / Cancer. 2008;44(4):557-64. https://doi.org/10.1016/j.ejca.2007.12.001

4. Castellsagué X, Sanjosé S, Aguado T, Louie KS, Bruni L, Muñoz J, et al, editors. HPV and cervical cancer in the world: 2007 report. Vaccine. 2007 [citado 30 jun 2017];25 Suppl 3:c1-26. Disponível em: http://www.hpvcentre.net/link_media/HPVReport2007.pdf

5. Cuzick J, Cadman L, Mesher D, Austin J, Ashdown-Barr L, Ho L, et al. Comparing the performance of six human papillomavirus tests in a screening population. Br J Cancer. 2013;108(4):908-13. https://doi.org/10.1038/bjc.2013.22

6. Damacena GN, Szwarcwald CL, Malta DC, Souza Júnior PRB, Vieira MLFP, Pereira CA, et al. O processo de desenvolvimento da Pesquisa Nacional de Saúde no Brasil, 2013. Epidemiol Serv Saude. 2015;24(2):197-206. https://doi.org/10.5123/S1679-49742015000200002

7. Demir ET, Ceyhan M, Simsek M, Gunduz T, Arlier S, Aytac R, et al. The prevalence of different HPV types in Turkish women with a normal Pap smear. J Med Virol. 2012;84(8):1242-7. https://doi.org/10.1002/jmv.23333

8. Dondog B, Clifford GM, Vaccarella S, Waterboer T, Unurjargal D, Avirmed D, et al. Human papillomavirus infection in Ulaanbaatar, Mongolia: a population-based study. Cancer Epidemiol Biomarkers Prev. 2008;17(7):1731-8. https://doi.org/10.1158/1055-9965.EPI-07-2796

9. Facina T. Estimativa 2014 - Incidência de Câncer no Brasil. Rev Bras Cancerol. 2014 [citado 30 jun 2017];60(1):63-4. Disponível em: http://www.inca.gov.br/rbc/n_60/v01/pdf/11-resenhaestimativa-2014-incidencia-de-cancer-no-brasil.pdf

10. Forman D, Bray F, Brewster DH, Gombe Mbalawa C, Kohler B, Piñeros M, et al, editors. Cancer incidence in five continents. Vol. X. Lyon: International Agency for Research on Cancer; 2013 [citado 19 jul 2013]. Disponível em: http://ci5.iarc.fr

11. Girianelli VR, Thuler LCS, Azevedo e Silva G. Prevalência de HPV em mulheres assistidas pela Estratégia Saúde da Família na Baixada Fluminense do Estado do Rio de Janeiro. Rev Bras Ginecol Obstet. 2010;32(1):39-46. https://doi.org/10.1590/S0100-72032010000100007

12. Girianelli VR, Gamarra CJ, Azevedo e Silva G. Os grandes contrastes na mortalidade por câncer do colo uterino e de mama no Brasil. Rev Saude Publica. 2014;48(3):459-67. https://doi.org/10.1590/S0034-8910.2014048005214

13. Levi JE, Longatto-Filho A, Eluf-Neto J, Rodrigues CL, Oliveira CM, Carloni AC, et al. Evaluation of HPV molecular tests in primary screening for cervical cancer in Brazil. Open J Obstet Ginecol. 2014;4(8):470-8. https://doi.org/10.4236/ojog.2014.48068.

14. Malta DC, Silva Jr JB. O plano de ações estratégicas para o enfrentamento das doenças crônicas não transmissíveis no Brasil e a definição das metas globais para o enfrentamento dessas doenças até 2025: uma revisão. Epidemiol Serv Saude. 2013;22(1):151-64. https://doi.org/10.5123/S1679-49742013000100016

15. Marçal JA, Gomes LTS. A prevenção do câncer do colo do útero realizada pelo enfermeiro na Estratégia Saúde da Família: revisão integrativa da literatura. Rev Eletron Acervo Saude. 2013 [citado 30 jun 2017];5(2):479-89. Disponível em: http://acervosaud.dominiotemporario.com/ doc/artigo_035.pdf

16. Novaes HMD, Soárez PC, Silva GA, Ayres A, Itria A, Rama CH, et al. Cost-effectiveness analysis of introducing universal human papillomavirus vaccination of girls aged 11 years into the National Immunization Program in Brazil. Vaccine. 2015;33 Suppl 1:A135-42. https://doi.org/10.1016/j.vaccine.2014.12.031

17. Núñez-Troconis J, Delgado M, González J, Mindiola R, Velásquez J, Conde B, et al. Prevalence and risk factors of human papillomavirus infection in asymptomatic women in a Venezuelan urban area. Invest Clin. 2009;50(2):203-12.

18. Pista A, Oliveira CF, Cunha MJ, Paixão MT, Real O; CLEOPATRE Portugal Study Group. Risk factors for human papillomavirus infection among women in Portugal: the CLEOPATRE Portugal Study. Int J Gynaecol Obstet. 2012;118(2):112-6. https://doi.org/10.1016/j.ijgo.2012.03.028

19. Rojas IQ. The cervical cancer prevention programme in Costa Rica. Ecancermedicalscience. 2015;9:578. https://doi.org/10.3332/ecancer.2015.578 
20. Ronco G, Dillner J, Elfström KM, Tunesi S, Snijders PJ, Arbyn M, et al. Efficacy of HPV-based screening for prevention of invasive cervical cancer: follow-up of four European randomized controlled trials. Lancet. 2014;383(9916):524-32. https://doi.org/10.1016/S0140-6736(13)62218-7

21. Sankaranarayanan R. Screening for cancer in low- and middle-income countries. Ann Glob Health. 2014;80(5):412-7. https://doi.org/10.1016/j.aogh.2014.09.014

22. Sarian LO, Derchain SFM, Bastos JFB. Métodos diagnósticos para o rastreamento do câncer do colo. Rev Bras Ginecol Obstet. 2010;32(8):363-7. https://doi.org/10.1590/S0100-72032010000800001

23. Sherpa ATL, Clifford GM, Vaccarella S, Shrestha S, Nygård M, Karki BS, et al. Human papillomavirus infection in women with and without cervical cancer in Nepal. Cancer Causes Control. 2010;21(3):323-30. https://doi.org/10.1007/s10552-009-9467-z

24. Shin HR, Lee DH, Herrero R, Smith JS, Vaccarella S, Hong SH, et al. Prevalence of human papillomavirus infection in women in Busan, South Korea. Int J Cancer. 2003;103(3):413-21. https://doi.org/10.1002/ijc.10825

25. Silva KC, Rosa MLG; Moyses N; Afonso LA; Oliveira LHS; Cavalcanti SMB. Risk factors associated with human papillomavirus infection in two populations from Rio de Janeiro, Brazil. Mem Inst Oswaldo Cruz. 2009;104(6):885-91. https://doi.org/10.1590/S0074-02762009000600011

26. Vaccarella S, Franceschi S, Herrero R, Muñoz N, Snijders PJ, Clifford GM, et al. Sexual behavior, condom use, and human papillomavirus: pooled analysis of the IARC human papillomavirus prevalence surveys. Cancer Epidemiol Biomarkers Prev. 2006;15(2):326-33. https://doi.org/10.1158/1055-9965.EPI-05-0577

27. Vaccarella S, Herrero R, Dai M, Snijders PJ, Meijer CJ, Thomas JO, et al. Reproductive factors, oral contraceptive use, and human papillomavirus infection: pooled analysis of the IARC HPV prevalence surveys. Cancer Epidemiol Biomarkers Prev. 2006;15(11):2148-53. https://doi.org/10.1158/1055-9965.EPI-06-0556

28. Vaccarella S, Herrero R, Snijders PJ, Dai M, Thomas JO, Hieu NT, et al. Smoking and human papillomavirus infection: pooled analysis of the International Agency for Research on Cancer HPV prevalence surveys. Int J Epidemiol. 2008;37(3):536-46. https://doi.org/10.1093/ije/dyn033

29. Vaccarella S, Lortet-Tieulent J, Plummer M, Franceschi S, Bray F. Worldwide trends in cervical cancer incidence: impact of screening against changes in disease risk factors. Eur J Cancer. 2013;49(15):3262-73. https://doi.org/10.1016/j.ejca.2013.04.024

30. Wentzensen N. Triage of HPV-positive women in cervical cancer screening. Lancet Oncol. 2013;14(2):107-9. https://doi.org/10.1016/S1470-2045(12)70568-5

31. Zorzi M, Del Mistro A, Farruggio A, Bartolomeis L, Frayle-Salamanca H, Baboci L, et al. Use of a high-risk human papillomavirus DNA test as the primary test in a cervical cancer screening programme: a population-based cohort study. BJOG. 2013;120(10):1260-7. https://doi.org/10.1111/1471-0528.12272

Financiamento: Edital Universal Conselho Nacional de Desenvolvimento Científico e Tecnológico (CNPq Processo 478949/2010-3).

Contribuição dos Autores: Concepção e planejamento do estudo: ARGA, GAS, MTBT,CJG. Coleta, análise e interpretação dos dados: MLSMM, KCDD, JEL, ARGA, GAS. Elaboração ou revisão do manuscrito: ARGA, GAS. Aprovação da versão final: ARGA,GAS. Responsabilidade pública pelo conteúdo do artigo: ARGA, GAS.

Conflito de Interesses: Os autores declaram não haver conflito de interesses. 